\title{
Chronic Narcotic Therapy for Patients with Chronic Non-malignant Pain
}

\author{
a report by \\ Gary W Jay
}

Board of Advisors, American Academy of Pain Management (AAPM), and

Medical Director, Headache and NeuroPain Care Institute

DOI:10.17925/ENR.2006.00.01.39

The use of chronic narcotics in the management of chronic non-malignant pain (CNMP) is an important but extremely controversial subject. Hardy noted in 1991 that "there is no place for opiates in the treatment of chronic benign pain". A year later, the American Pain Society (APS) survey of its physician members indicated that opioids are probably underutilised in CNMP.

Types of Pain

CNMP is a biological-psychological-sociological phenomenon. All aspects of these problem areas must be appropriately diagnosed and treated. To not do so will prevent a good outcome from treatment - secondary to lack of amelioration of pain and inability of the patient to regain function. A major differentiating factor between acute-pain patients and those with CNMP is that the latter typically do not have significant physiological objective findings on examination that correlate to the patients' complaints. Those that have objective findings may not appear to have findings that would support the degree of their subjective complaints of chronic, severe pain. These patients are numerous - statistics indicate that the approximate total number of such patients in the US is at least 70 million. The most common forms of CNMP include:

- myofascial pain syndromes;

- fibromyalgia;

- neuropathic (nerve-related) pain;

- radiculopathies (chronic central nerve impingement by a disc in the spinal column);

- failed back syndromes (continued pain after one or more neck or mid or low back surgeries);

- complex regional pain syndrome (formerly called reflex sympathetic dystrophy); and

- sympathetically maintained pain - secondary to autonomic nervous system dysfunction, arthritis and chronic headache.

The biological-psychological-sociological phenomenon that encompasses CNMP is most time-, cost- and clinically effectively treated within the framework of an interdisciplinary pain management programme. Such a programme consists of the physician medical director (typically a neurologist or physiatrist), who makes diagnoses and deals with medical management, including neuropharmacological care; nurses, who act as both internal case managers and educators; psychologists, who deal with cognitivebehavioural therapy, biofeedback neuromuscular reeducation and other aspects of individual and group treatment; and rehabilitation professionals, including physical therapists and occupational therapists. Other members of a pain management team may include social workers, vocational specialists and alternative medicine specialists. Published research, including evaluated evidence-based medicine with metaanalyses, has demonstrated the clinical effectiveness, along with the time- and cost-effectiveness, of these programmes. Unfortunately, very few chronic-pain patients, possibly up to $6 \%$, obtain treatment in an interdisciplinary pain medicine programme. For the treatment of certain chronic pain conditions, neurostimulation may be the appropriate approach (see separate text box).

The typical pain patient is seen first frequently by their primary care physicians, a generalist or family physician. Tests are performed, probably including magnetic resonance imaging (MRI) and/or computed axial tomography (CAT) scans. If these clinicians are unable to help the patients, especially if any of these tests are positive, they are sent for a consultation to an orthopaedist or neurosurgeon, and more tests may be performed. It should be remembered that many people without complaints of pain have an 'abnormal MRI or CAT scan' of the cervical or lumbosacral spine. If surgery is not performed, the patient is typically sent to an interventional anaesthesiologist, who will perform multiple treatments (epidural steroid injections, facet/medial branch nerve blocks and radiofrequency neurolysis/rhizotomies). Often, these patients receive only a temporary decrement in their pain, which will soon return to baseline levels. The interventionalists may place the patients on narcotic pain medication or send them back to their referring physician for such pain medications.

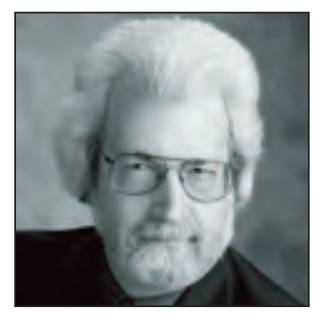

Gary W Jay is on the Board of Advisors of the American Academy of Pain Management (AAPM) and Medical Director of the Headache and NeuroPain Care Institute in Tamarac, Florida. He is Past President of the Florida Academy of Pain Medicine (FAPM) and is its Executive Medical Director. Dr Jay is the author of three textbooks, with a fourth being written, and more than 36 published medical textbook chapters and peer-reviewed journal articles. He is a frequent lecturer on headache, pain of all types and the appropriate usage of narcotic analgesics. 
Electric stimulation has a long history in medicine for treating various ailments. Neurostimulation is the application of precise targeted electrical stimulation on nociceptive pathways. Beyond the application of electrodes on the skin, such as in transcutaneous electrical nerve stimulation (TENS), electrodes have been applied directly to nociceptive pathways.

The nociceptive pathways are made up of tracts in the central and peripheral nervous systems (CNS and PNS). The CNS includes nociceptive pathways in the spinal cord and brain, specifically the dorsal roots, dorsal ganglion, spinothalamic tracts and all ascending neural tracts to the cerebrum. The PNS includes pathways outside the spinal cord, specifically various plexuses and peripheral nerves.

How Neurostimulation Controls Pain

Neurostimulation delivers low-voltage electrical stimulation to the spinal cord or targeted peripheral nerve to block the sensation of pain. One theory, the Gate Control Theory of pain, proposes that neurostimulation activates the body's pain inhibitory system. According to this theory,

Using Narcotics Appropriately

State and federal clinical practice guidelines do indicate that it is appropriate to alleviate pain, and that the use of pain medications to do so is legal. A new set of 'frequently asked questions' was released by the US Drug Enforcement Agency (DEA) and pain specialists from the University of Wisconsin, in August 2004, the purpose of which was to indicate that physicians "cannot be arrested for properly prescribing narcotic pain killers that are the best treatment for millions of suffering patients". The Joint Commission for Accreditation of Health Care Organizations (JCAHO) has determined that pain is the 'fifth vital sign' and mandated significant changes in hospital facilities to deal with this problem.

Undertreatment with opioid pain medication is becoming all too common for an estimated $40-70 \%$ of patients with chronic, intractable non-malignant pain.

Medically, there are significant adverse effects of undertreated pain:

- Physical

- increased pulse, blood pressure and respiration;

- increased risk of cardiac event in patients so predisposed;

- increased risk of atelectasis and pneumonia; there is a gate in the spinal cord that controls the flow of noxious pain signals to the brain. The theory suggests that the body can inhibit these pain signals or 'close the gate' by activating certain nonnoxious nerve fibres in the dorsal horn of the spinal cord. The neurostimulation system, implanted in the epidural space, stimulates these pain-inhibiting nerve fibres, masking the sensation of pain with a tingling sensation (paraesthesia).

Neurostimulation may be the appropriate treatment for the management of certain chronic pain conditions, including failed back syndrome (FBS), complex regional pain syndrome (CRPS) and arachnoiditis.

\section{Conclusion}

Neurostimulation of the CNS and PNS plays a vital role in the treatment of various difficult pain conditions, such as complex regional pain syndrome. Until treatments that truly eliminate pain are developed, neurostimulation can play a major role in improving the quality of life for pain patients. Neurostimulation systems do not damage neural pathways and can be removed when curative therapy becomes available.

- decreased tissue oxygenation, leading to muscle breakdown, poor healing and weakness;

- decreased activity, mobility leading to decreased recovery secondary to limited ambulation; and

- increased risk of thromboembolic events.

- Psychological

- depression and anxiety disorders;

- sleep deprivation; and

- anorexia.

- Immunological

- decreased immune response secondary to decreased nature killer cells.

- Socioeconomic

- decreased productivity and loss of work;

- increased use of healthcare resources; and

- familial breakdown.

Treatment of the CNMP patient with only narcotics is problematic and most often leads to failure. The most appropriate treatment is within an interdisciplinary pain management programme. An important issue here is that part of the typical interdisciplinary programme is the use and subsequent weaning off of chronic opioid medications, as tolerated by the patient and reflected by their continued and improved functionality. The use of opioids in the CNMP patient does not have a routine, 


\section{MEDTRONIC PAIN THERAPIES}

Available drug therapies are effective in $50 \%$ of neuropathic pain patients. What about the other $50 \%$ ?

Neurostimulation with Medtronic Pain Therapies

The only neurostimulation systems clinically proven to:

- Provide $50-60 \%$ pain relief longterm in $>60 \%$ of patients

- Improve QoL at an excellent side effect profile

Which one of your patients can benefit?
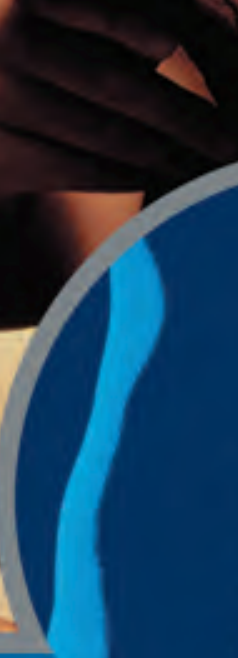

Craig, Orthopedic Surgeon and CRPS patient, benefits from Medtronic Pain Therapies

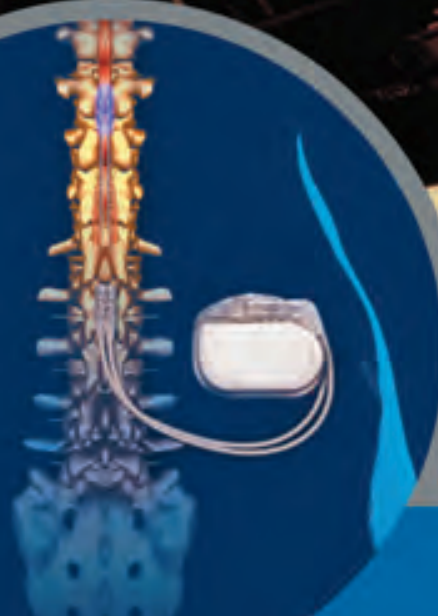

Medtronic Pain Therapies: Less Pain for a Better Life 
non-individualised answer. Clinically, many patients with CNMP with a very poor quality of life can improve their function with the use of time-release (around-the-clock) opioid pain medications. On the other hand, some patients may develop decreased functionality with chronic opioids. Therefore, function is the most important issue when dealing with pain patients and chronic opioid medications. If these patients are not showing an improved functionality on these medications, they may need to be stopped.

Most importantly, prior to the use of chronic opioid medications, the CNMP patients must have received all conservative and/or appropriate surgical treatment and failed it, meaning that their pain was not ameliorated, and their functionality continued to be low or show further decline. For patients who have had and failed all appropriate treatment, the use of chronic narcotics may certainly be appropriate on an individual basis. There are several tenets that should be followed. First, these patients should receive longacting opioids on an around-the-clock basis to maintain an acceptable level of comfort. These medications provide a relatively flat dose-response curve that engenders effective levels of analgesia without the peaks and valleys seen with short-acting pain medications, and thus provide less risk for potential drug abuse.

There are four basic time-release medications:

- Duragesic Patches (Janssen) - a percutaneous fentenyl used on the skin for, most typically, 72 hours at a time. This medication also enables the patient to stop taking painkillers several times a day, helping to extinguish a medication-related behaviour.

- Morphine sulfate - this exists in different timerelease formulations - MS Contin (Purdue Pharma) and Kadian (Alpharma, Inc.) are both formulations to be given, most typically every 12 hours, and Avinza (Ligand Pharmaceuticals) is a once-a-day preparation.

- Oxycontin (Purdue Pharma) - this is a timerelease oxycodone, typically taken every 12 hours. It was this medication, along with its nickname 'Hillbilly Heroin' and multiple stories of drug abuse and drug diversion and addiction that brought the current crisis regarding the use of these medications to a head.

- Methadone (generic) - this is a very old medication, developed in the mid 20th century. This medication has a long half-life - it is not a timerelease medication. It may be given every eight to 12 hours. The difficulty in its use is two-fold - poor understanding by the physician of its clinical attributes and use, and the fact that it is also used in specific government-approved heroin/opioid detoxification programmes. Some pharmacies insist on having the words 'for pain' on the prescription, or they will not fill it in - another institutional problem for these patients is the stigma.

Even with a time-release medication, the CNMP patients on occasion need to be given a 'breakthrough pain' medication, typically a short-acting, immediaterelease opioid that may be needed to lower nociceptive pain brought about by an acute exacerbation of pain secondary to any number of factors, such as overactivity. The typical immediate-release medications used for breakthrough pain include:

- Darvocet (N-100) - propoxyphene, a mild narcotic that can have significant side effects in the elderly;

- Ultram, Ultracet (Tramadol, with or without acetaminophen) - a medication that stimulates the mu-opioid receptors, as well as serotonin and norepinephrin;

- Vicodin (Abbott Pharmaceutical) - hydrocodone and acetaminophen given every four to six hours for breakthrough pain;

- Lorcet (Forest Pharmaceuticals), Lortab (UCB Pharma, Inc.), Norco (Watson Pharmaceuticals) hydrocodone and acetaminophen, to be used every four to six hours for breakthrough pain. Norco has the smallest dosages of acetaminophen, making the acetaminophen load lowest, therefore least hepatotoxic, depending on the number utilised each day;

- Percocet (Endo Pharmaceuticals) - oxycodone and acetaminophen, given every four to six hours for breakthrough pain;

- Roxicodone (Roxane Laboratories, Inc.) - oxycodone without acetaminophen, given every four to six hours for breakthrough pain; and

- Actiq (Cephalon, Inc.) - fentanyl oral transmucosal, an oralette or 'lollypop' on a stick - allowed to dissolve in the mouth, with medication entering the system transmucosally, to be used every four to six hours for severe breakthrough pain.

The use of immediate-release narcotics given with acetaminophen and/or a non-steroidal antiinflammatory medication must be looked at carefully secondary to possible hepato- and nephrotoxicity.

CNMP patients should be seen monthly. If an escalation of the amount of breakthrough pain 
medication is seen, this may indicate a need to increase the time-release chronic narcotics. In routine practice, the CNMP patients should be given a pain medication agreement, which indicates the possible side effects of narcotics (including sedation, nausea, vomiting, itching, loss of sexual function and immunological problems, among others). Also, the patient must agree that only one physician will provide his/her narcotic pain medications, and the prescriptions will be taken to one (listed) pharmacy. Urine or blood tests may be performed at any time, and if an untoward substance (i.e. cocaine or narcotics not prescribed by the pain specialist) is found in the patient's urine or blood, or if their blood/urine level of the prescribed pain medication is very inappropriate, the physician may wean the patient off their opioid medications and discharge them. Other reasons for tapering and ending opioid maintenance include evidence of opioid hoarding, the patient obtaining pain medications from other prescribers, the patient obtaining drugs from others (diversion) and uncontrolled dose escalation or other aberrant behaviours (loss of the medication, reports of stolen medications without a proper police report and a patient frequently calling in requesting medications earlier than should be indicated after being given a one-month drug prescription). A past history of substance abuse may be considered a relative contraindication for the use of chronic opioids.

Barriers to the Use of Opioids

There are patient and physician barriers to appropriate use of opioids - fears of addiction, medication dependence, and drug tolerance, with frequent lack of understanding of the differences between these issues. Physicians are often reluctant to prescribe opioids due to:

- an inadequate understanding of pain management principles;

- inability to appropriately assess a patient's pain;

- fear/concern about regulation of controlled substances;

- fear that giving pain medication to one patient would make the physician a target of the DEA as well as of other patients wanting/needing pain medications;

- fears of patient addiction and other problems leading to liability;

- concern about patients becoming tolerant to opioids, needing higher dosages and needing them for extended periods of time (years); and

- concern about side effects of opioids.

Patient barriers to appropriate opioid use include:

- fear that pain means that a disease is worse;
- concern that talking about pain would prevent a physician from dealing with a significant underlying disease;

- wanting to be a good patient and not alienate the physician by reporting pain;

- concerns about developing tolerance or addiction to pain medications;

- fear of showing 'weakness'; and

- embarrassment to go to the pharmacy for these drugs, as well as further embarrassment that he/she may be construed a 'drug addict' even though he/ she has no aberrant drug-related behaviour and has a physiological reason for his/her pain.

Systemic/institutional barriers include:

- restrictive regulation of controlled substances;

- poor access to treatment;

- poor access to pain management specialists; and

- poor insurance:

- the most appropriate treatment will not be reimbursed;

- the most appropriate treatment is too costly for the patient;

- lack of rehabilitation benefits - 'bad insurance'; or

- inability to obtain any rehabilitation, or even just physical therapy or psychological care, as they are far more expensive than pain medications (an hour of physical therapy may cost US $\$ 150-200$, while a single generic Tylenol \#4 tablet - one grain of codeine - costs only pennies).

Other barriers to pain relief are the facts that pharmacies do not stock adequate and/or appropriate opioids and that there is no continuity in patient care.

\section{Sum mary}

The complexity of this subject is great, even though the medical aspects are fairly straightforward. It is the multitudes of other problems and barriers to appropriate pain management and opioid usage that make this problem so complex.

When considering that the clinical definition of pain is simple - what ever the patient says it is - it is then up to the clinician to determine exactly what the patient means, utilising a history, examination and any necessary tests. Appropriate treatment should then be rendered. The utilisation of chronic opioid analgesics is one very important treatment modality, which, when used appropriately, may help to improve patients' function and ameliorate their pain.

A version of this article containing references can be found in the Reference Section on the website supporting this briefing (www.touchbriefings.com). 